Article

\title{
Laser-plasma and self-absorption measurements with applications to analysis of atomic and molecular stellar astrophysics spectra
}

\author{
Christian G. Parigger ${ }^{1, *, \dagger} \mathbb{1}$, Christopher M. Helstern ${ }^{1, \dagger}$ and Ghaneshwar Gautam ${ }^{2, \dagger}$ \\ 1 Physics and Astronomy Department, University of Tennessee, University of Tennessee Space Institute, \\ Center for Laser Applications, 411 B.H. Goethert Pkwy, Tullahoma, TN 37388, USA; \\ Chris.Helstern@gmail.com \\ 2 Mathematics and Science Department, Fort Peck Community College, 605 Indian Avenue, Poplar, MT 59255, \\ USA; ggautam@fpcc.edu \\ * Correspondence: cparigge@tennessee.edu; Tel.: +1-931-841-5690 \\ + These authors contributed equally to this work.
}

\begin{abstract}
This work discusses laboratory measurements of atomic and diatomic molecular species in laser-plasma generated in gases. Noticeable self-absorption of the Balmer series hydrogen alpha line occurs for electron densities of the order of one tenth of standard ambient temperature and pressure density. Emission spectra of selected diatomic molecules in air or specific gaseous mixtures at or near atmospheric pressure reveal minimal plasma re-absorption. Abel inversion of the plasma in selected gases and gas mixtures confirm expansion dynamics that unravel regions of atomic and molecular species of different electron temperature and density. Time resolved spectroscopy diagnoses self-absorption of hydrogen alpha and hydrogen beta lines in ultra-high pure hydrogen gas. Radiation from a Nd:YAG laser device induces micro-plasma for pulse widths in the range of $6 \mathrm{~ns}$ to $14 \mathrm{~ns}$, energies in the range of $100 \mathrm{~mJ}$ to $800 \mathrm{~mJ}$, and for peak irradiance of the order 1 to 10 $\mathrm{TW} / \mathrm{cm}^{2}$. Atomic line profiles yield electron density and temperature from fitting of line profiles to wavelength and sensitivity corrected spectral radiance data. Analysis of measured diatomic emission data yields excitation temperature of primarily molecular recombination spectra. Applications of the laboratory experiments extend to investigations of stellar astrophysics white dwarf spectra.
\end{abstract}

Keywords: atomic and molecular spectroscopy; time-resolved spectroscopy; laser plasma; laser-induced optical breakdown; stellar astrophysics spectra; white dwarf stars; hydrogen; carbon Swan spectra

\section{Introduction}

Measurements of optical spectra from stellar astrophysical objects usually provide essential data for the determination of star-atmospheres and astrophysical phenomena [1]. White dwarf stars Sirius $\mathrm{B}$ and Procyon B, companions to Sirius and Procyon, reveal radiation temperatures of $26 \mathrm{kK}$ and 8 $\mathrm{kK}$, respectively [2]. The optical spectra of Sirius B show exclusively atomic, hydrogen Balmer series absorption spectra in the range of $400 \mathrm{~nm}$ to $700 \mathrm{~nm}$. In turn, Procyon B reveals molecular, carbon Swan absorption spectra. This work also presents analysis of astrophysical absorption spectra from the Montreal data-base [2] and selected hydrogen absorption spectra from the Keck observatory archieves (KOA) [3]. Application of well-established plasma spectroscopy [4] is instrumental for laboratory laser micro-plasma experiments for simulation of astrophysical conditions, in particular, those of white dwarfs.

This work presents characterization of gaseous hydrogen micro-plasma from the first four Balmer series members, $\mathrm{H}_{\alpha}, \mathrm{H}_{\beta}, \mathrm{H}_{\gamma}$, and $\mathrm{H}_{\delta}$ lines. Previously communicated work primarily were results from $\mathrm{H}_{\alpha}$ and $\mathrm{H}_{\beta}$ lines $[5,6]$. The electron density, $\mathrm{N}_{\mathrm{e}}$, of interest ranges from $10^{16} \mathrm{~cm}^{-3}$ to $10^{19} \mathrm{~cm}^{-3}$ for laser-plasma emission experiments. The laboratory measurements utilize time-resolved spectroscopy 
[7], but addition in the analysis of these data is necessary for interpretation of recorded white dwarf spectral line shapes. Discussion is included of the spectral appearance of the first four Balmer series as these merge with the free-electron background radiation, i.e., near the Inglis-Teller limit [8].

Self-absorption phenomena in $\mathrm{H}_{\alpha}$ laser-plasma experiments become important for electron densities, $\mathrm{N}_{\mathrm{e}}$, of the order of $\eta=\mathrm{N}_{\mathrm{e}} / n_{0}=0.1 \mathrm{amg}$ [9], where $\mathrm{n}_{0}=268.68 \times 10^{17} \mathrm{~cm}^{-3}$ at standard ambient temperature and pressure (SATP). Selected white dwarf spectral data display absorption profiles from atmospheres with electron densities of $\sim 1-5 \times 10^{17} \mathrm{~cm}^{-3}$, or $\eta=0.004-0.02 \mathrm{amg}$, consequently, the $\mathrm{H}_{\beta}$ line appears most suited for diagnosis of such electron density. The $\mathrm{H}_{\beta}$ line allows one to determine electron density from full-width at half-maximum, peak-separation, and central dip-shifts $[5,6]$ for $\mathrm{N}_{\mathrm{e}}$ in the range $2-20 \times 10^{17} \mathrm{~cm}^{-3}$.

Comparisons of spectra from laboratory plasma and astrophysical white dwarf stars has been content of research and documented communications for several decades or about 50 years. Noteworthy are the discussions from the 1970's regarding the Stark-effect caused redshifts [10-12] that may be larger than the gravitational- or Einstein- redshifts [13], casting doubt in the determination of the white dwarf ratio of mass over radius. The gravitational redshift can be understood with the Schwarzschild metric [15]. Radiative-transfer and error margin considerations [16] conclude that the pressure shifts are well within error margins of the determined gravitational shifts. A recent re-discussion in 2015 [17] indicates that line profiles from white dwarf stars are composed of contributions from different atmosphere layers, and the recorded integrated spectra mask the Stark-effect or pressure shifts.

\section{Materials and Methods}

The experimental arrangement for laboratory measurements of laser-induced plasma [6] includes a Q-switched Nd:YAG laser device typically generating 6 ns laser pulses with an energy of $150 \mathrm{~mJ}$ per pulse at the fundamental wavelength of $1064 \mathrm{~nm}$. Radiation is tightly focused to a spot size of the order of $10 \mu \mathrm{m}$ to achieve a peak irradiance above $1 \mathrm{TW} / \mathrm{cm}^{2}$, or well above optical breakdown of SATP laboratory air and gaseous hydrogen of the order of one atmosphere. The emanating light from the micro-plasma is recorded with a Czerny-Turner type spectrometer that shows a resolution of $0.02 \mathrm{~nm}$ when using a 3600 groves $/ \mathrm{mm}$ holographic grating and an intensified diode array or two-dimensional detector for time-resolved spectroscopy. Figure 1 illustrates the experimental schematic.

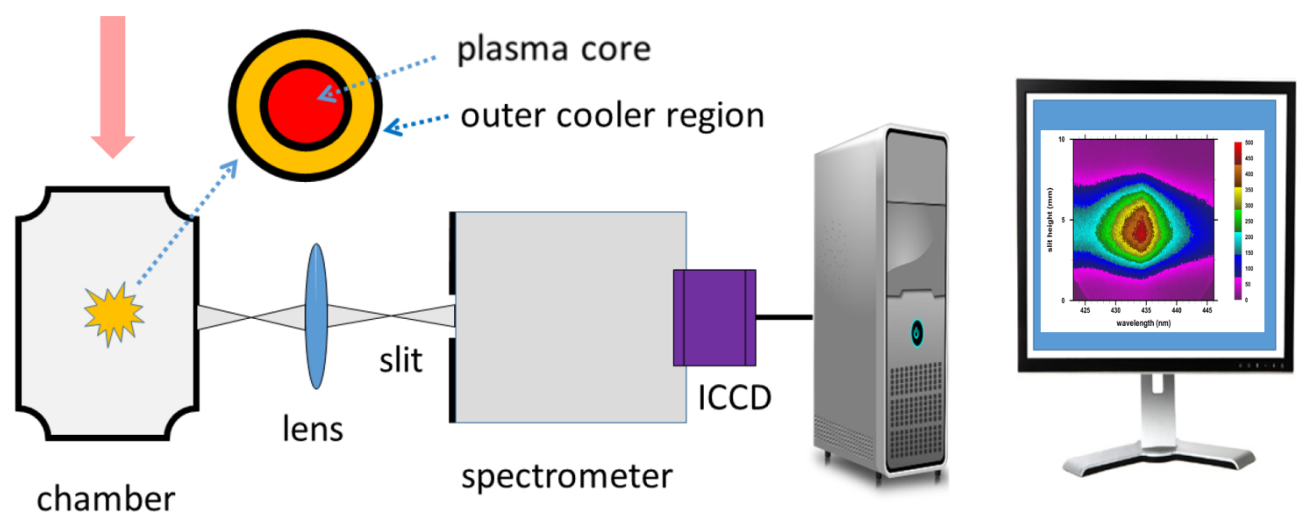

data recording and processing

Figure 1. Optical breakdown plasma is generated by focusing the laser beam from the top and parallel to the slit. Time-resolved 1:1 images are recorded with the ICCD.

The astrophysical data sets are preferably recorded with a high resolution spectrometer, for instance the so-called HIRES instrument at the Keck observatory that utilizes an echelle grating. The 
spectral resolving power, $R=\lambda / \Delta \lambda$, i.e., the ratio of wavelength, $\lambda$, and of the spectral resolution, $\Delta \lambda$, typically is of the order of 40,000 .

At $\mathrm{H}_{\beta}$, the spectral resolution equals $\Delta \lambda=0.012 \mathrm{~nm}$. In terms of the average gravitational redshift, $\mathrm{vg}_{\mathrm{g}}=\mathrm{c} \Delta \lambda / \lambda$, with $\mathrm{c}$ the speed of light, the velocity resolution amounts to $7.5 \mathrm{~km} / \mathrm{s}$. For comparison, the Sirius B gravitational redshift $[13,14]$ is $89 \pm 16 \mathrm{~km} / \mathrm{s}$. In other words, the Sirius B redshift equals $\Delta \lambda_{\text {Sirius B }}=0.14 \mathrm{~nm}$.

From the gravitational shift [18], $\mathrm{v}_{\mathrm{g}}=0.64 \mathrm{M} / \mathrm{R}$, with $\mathrm{M}$ and $\mathrm{R}$ denoting the white dwarf (WD) mass and radius in solar units, respectively, and using thermodynamic cooling models, the WD parameters are inferred, namely local gravitational constant, $\mathrm{g}$, temperature, $\mathrm{T}$, mass, $\mathrm{M}$, and radius, $\mathrm{R}$. Typical values for WD's with a hydrogen atmosphere $\mathrm{g}, \mathrm{T}, \mathrm{M}$, and $\mathrm{R}$ amount to $10^{6} \mathrm{~m} / \mathrm{s}^{2}, 30 \mathrm{kK}$, mass of the sun, and size of the earth, respectively.

\section{Laboratory results}

Figures 2 and 3 illustrate recent measurements of $\mathrm{H}_{\delta}$ and $\mathrm{H}_{\gamma}$ lines of laser plasmas in hydrogen gas. The figures display the average of 100 individually accumulated ICCD images. In these experiments, the laser beam propagates parallel to the slit, from top to bottom as indicated in the experimental schematic, see Fig. 1. The recorded data are corrected for detector background and wavelength sensitivity. Wavelength calibration is accomplished with standard pen-ray light sources. The displayed image values represent spectral radiance in arbitrary units.

(a)

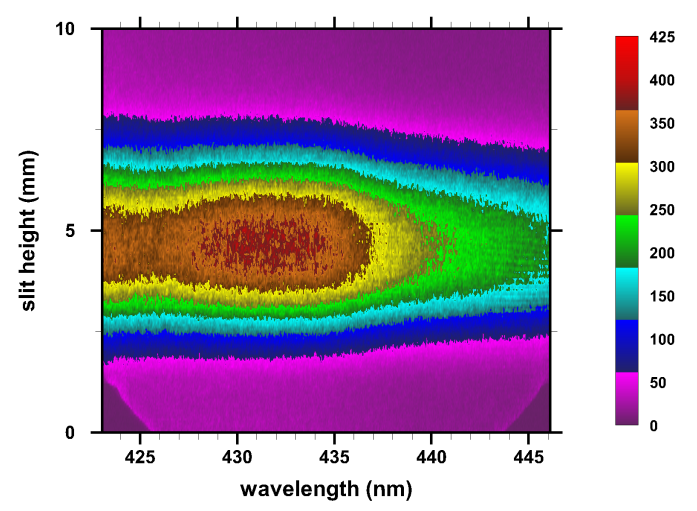

(c)

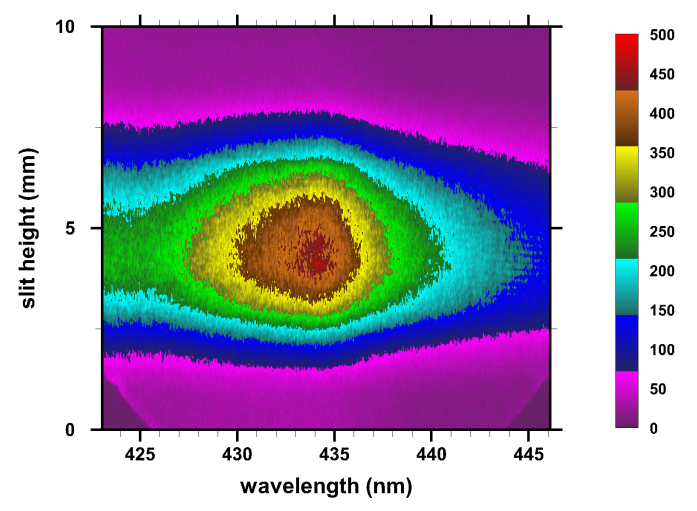

(b)

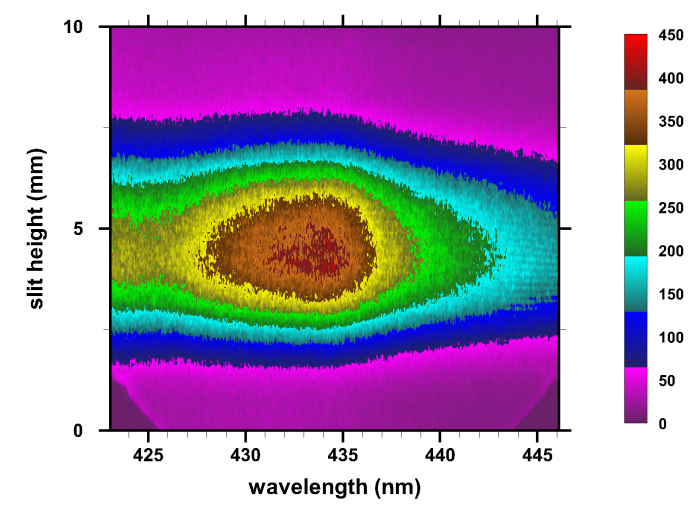

(d)

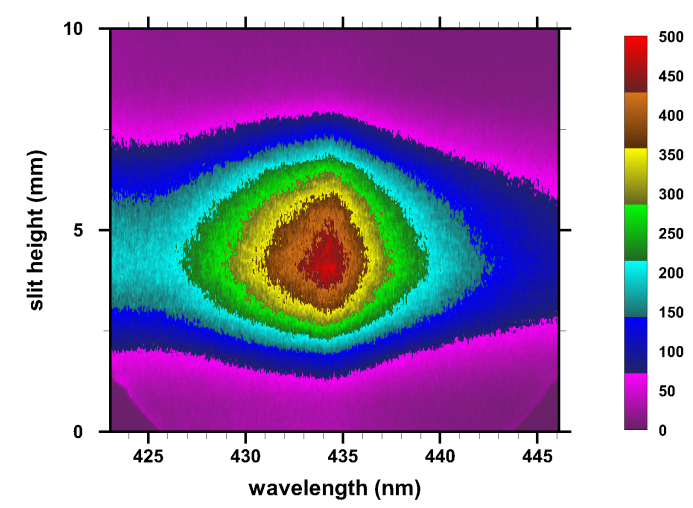

Figure 2. Recorded $\mathrm{H}_{\gamma}$ spectra along the slit for time delay of (a) $100 \mathrm{~ns}$, (b) $150 \mathrm{~ns}$, (c) $200 \mathrm{ns,} \mathrm{and} \mathrm{(d)}$ 275 ns. 
(a)

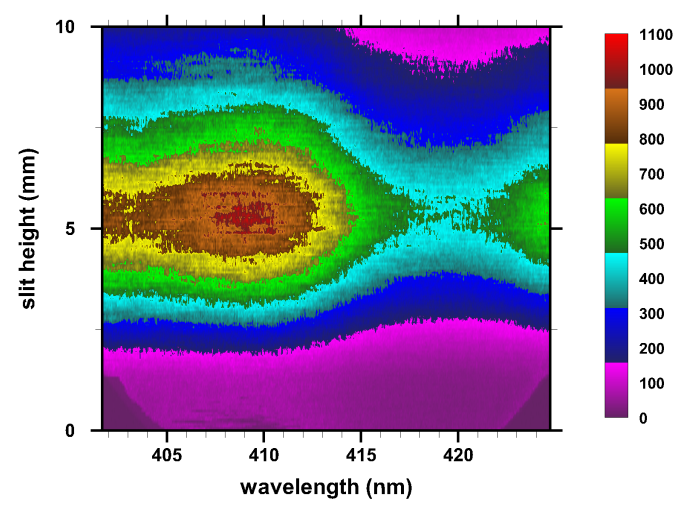

(c)

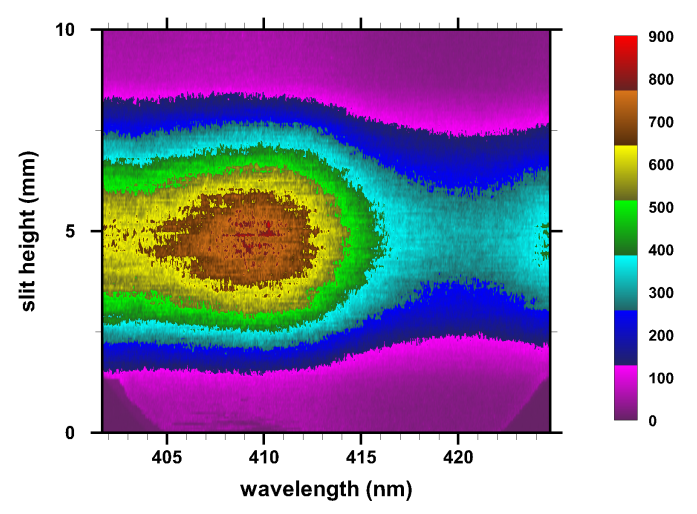

(b)

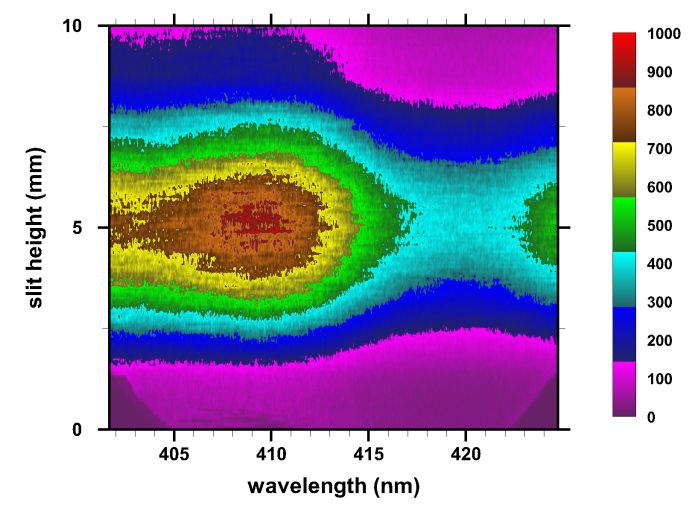

(d)

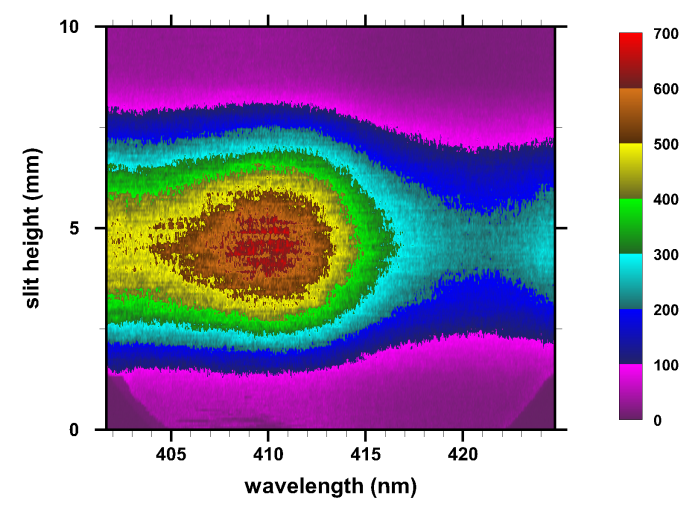

Figure 3. Recorded $\mathrm{H}_{\delta}$ spectra along the slit for time delay of (a) $100 \mathrm{~ns}$, (b) $150 \mathrm{~ns}$, (c) $200 \mathrm{~ns}$, and (d) 275 ns.

Fig. 2(a) displays $\mathrm{H}_{\gamma}$ (at $434.07 \mathrm{~nm}$ ) at the low-wavelength side of the image, background contributions from $\mathrm{H}_{\delta}$ (at $410.17 \mathrm{~nm}$ ) and contributions from free-electron radiation. Fig. 2(d) indicates diminished background contributions due to $\mathrm{H}_{\delta}$. Analogously, Fig. 3(a) shows $\mathrm{H}_{\delta}$ with contributions from $\mathrm{H}_{\gamma}$ at the high wavelength side of the image. On the low wavelength side but outside the recorded spectral window, the indicated contributions are likely from $\mathrm{H}_{\epsilon}$ (at $397.01 \mathrm{~nm}$ ) and are noticeable for all time delays in Fig. 3. The full-width at half-maxima are extracted from the data for determination of electron densities as a function of time delay.

Results for the $\mathrm{H}_{\beta}$ and $\mathrm{H}_{\alpha}$ and analysis were presented previously [5,6]. Electron densities from $\mathrm{H}_{\beta}$ agree with those from $\mathrm{H}_{\alpha}[5,6]$. Averages of the spatially resolved data along the slit yield spectra that appear similar to those recorded with a linear diode array [7]. Previously recorded $\mathrm{H}_{\alpha}$ and $\mathrm{H}_{\beta}$ experiments using a photomultiplier while scanning the spectrometer [7] debate comparisons of the standard [19] with the advanced Stark-broadening [22] theory.

The recorded $\mathrm{H}_{\gamma}$ and $\mathrm{H}_{\delta}$ maps reveal expected variations along the slit dimension. This work reports analysis of the spectra displayed in Figures 2 and 3. Published line shapes [23] are utilized for determination of electron density. The $\mathrm{H}_{\gamma}$ and $\mathrm{H}_{\delta}$ tables [23] are only available up to electron densities of $10^{17} \mathrm{~cm}^{-3}$ with temperatures up to $20 \mathrm{kK}$, but the tables usually show a weak temperature dependence. Both $\mathrm{H}_{\beta}$ and $\mathrm{H}_{\delta}$ show the typical dip at the center wavelength due to absence of the Stark component there. 
Analysis of the recorded spectra encompasses determination of temperature from the integrated line-to-continnuum ratios. Electron density measurements utilize full-width at half maximum (FWHM) and for $\mathrm{H}_{\beta}$ and $\mathrm{H}_{\gamma}$ peak-separation. However, the $\mathrm{H}_{\gamma}$ peak separation method is applied in analysis of experiments in 0.136-atm:0.136-atm $\mathrm{H}_{2}: \mathrm{N}_{2}$ gas mixtures (not reported here) that show electron densities in the range of $0.1-1 \times 10^{17} \mathrm{~cm}^{-3}$ for time delays longer than those for the $0.75-\mathrm{atm} \mathrm{H}_{2}$ experiments addressed in this work.

Figure 4 illustrates the line-to-continuum ratios and corresponding temperatures. The theoretical ratios of integrated line to 10-nm continuum ratios are recalculated [19], and the experimental data are added with error bars. The results for $\mathrm{H}_{\gamma}$ and $\mathrm{H}_{\delta}$ agree with previously communicated $\mathrm{H}_{\alpha}$ and $\mathrm{H}_{\beta}$ results [5]. Figs. 5(a) and 5(b) show Boltzmann plots [20,21] and inferred temperature for time delays of $275 \mathrm{~ns}$ and $150 \mathrm{~ns}$, respectively. The error bars reflect uncertainties in determination of the baseline. Temperatures from line-to-continuum ratios and Boltzmann plots agree.

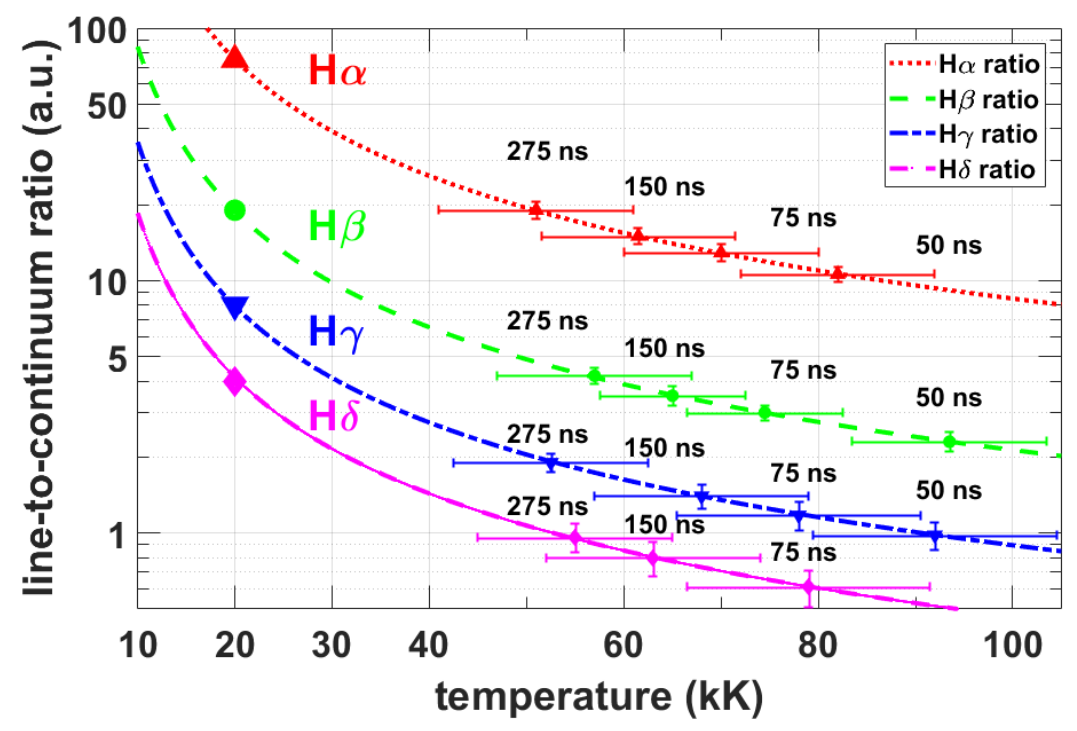

Figure 4. Computed and measured line-to-continuum ratios for selected time delays. Computed ratios of $75,19,8$, and 4 are highlighted for $\mathrm{H}_{\alpha}, \mathrm{H}_{\beta}, \mathrm{H}_{\gamma}$, and $\mathrm{H}_{\delta}$, respectively, at $20 \mathrm{kK}$.

(a)

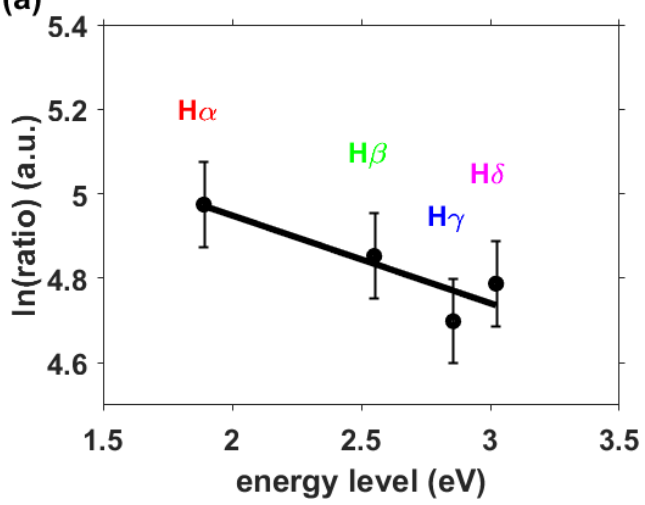

(b)

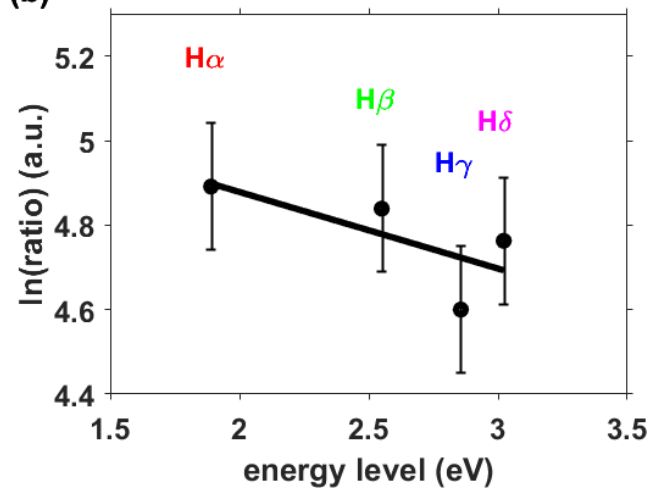

Figure 5. Boltzmann plots. (a) $\mathrm{T}=55 \mathrm{kK}$, time delay: $275 \mathrm{~ns}$; (b) $\mathrm{T}=64 \mathrm{kK}$, time delay: $150 \mathrm{~ns}$.

The Boltzmann plot utilizes the natural logarithm of the Boltzmann distribution for determination of the temperature,

$$
\ln \left\{\frac{\mathrm{I}_{\mathrm{ul}} \lambda_{\mathrm{ul}}}{\mathrm{g}_{\mathrm{u}} \mathrm{A}_{\mathrm{ul}}}\right\}=-\left\{\frac{\mathrm{E}_{\mathrm{u}}[\mathrm{eV}]}{\mathrm{T}[\mathrm{eV}]}\right\}+\ln \mathrm{C},
$$


where the integrated line intensities, $\mathrm{I}_{\mathrm{ul}}$, are determined in the experiment. The wavelengths, $\lambda_{\mathrm{ul}}$, are $656.28 \mathrm{~nm}, 486.14 \mathrm{~nm}, 434.05 \mathrm{~nm}$, and $410.17 \mathrm{~nm}$, with degeneracies, $\mathrm{g}_{\mathrm{u}}, 18,32,50$, and 72 . Transition probabilities, $\mathrm{A}_{\mathrm{ul}}$ are $4.410 \times 10^{7} \mathrm{~s}^{-1}, 0.8419 \times 10^{7} \mathrm{~s}^{-1}, 0.2530 \times 10^{7} \mathrm{~s}^{-1}$, and $0.09732 \times 10^{7} \mathrm{~s}^{-1}$. The excitation energies, $\mathrm{E}_{\mathrm{u}}$, are $1.89 \mathrm{eV}, 2.55 \mathrm{eV}, 2.856 \mathrm{eV}$, and $3.022 \mathrm{eV}$ for $\mathrm{H}_{\alpha}, \mathrm{H}_{\beta}, \mathrm{H}_{\gamma}, \mathrm{H}_{\delta}$, respectively. The constant, $\ln \mathrm{C}$, is not used - only the slope determines the temperature. Fig. 5 displays $\ln \{$ ratio $\}=$ $\ln \left\{\frac{\mathrm{I}_{\mathrm{ul}} \lambda_{\mathrm{ul}}}{\mathrm{g}_{\mathrm{u}} \mathrm{A}_{\mathrm{ul}}}\right\}$ versus $\mathrm{E}_{\mathrm{u}}$.

There are expected density variations across the slit height, but the analysis evaluates averages of the displayed spectra. For practical reasons, Lorentzian fitting is applied to find the full-width at half-maximum (FWHM) of the experimental spectra. From log-log fitting of $\mathrm{H}_{\gamma}$ and $\mathrm{H}_{\delta}$ tables in the range of $0.1-1 \times 10^{17} \mathrm{~cm}^{-3}$, one obtains for $\mathrm{H}_{\gamma} \mathrm{FWHM}, \Delta \lambda_{\gamma}$,

$$
\Delta \lambda_{\gamma}[\mathrm{nm}]=6.0\left(\frac{\mathrm{N}_{\mathrm{e}}\left[\mathrm{cm}^{-3}\right]}{10^{17}}\right)^{0.72}
$$

and for $\mathrm{H}_{\delta} \mathrm{FWHM}, \Delta \lambda_{\delta}$,

$$
\Delta \lambda_{\delta}[\mathrm{nm}]=10\left(\frac{\mathrm{N}_{\mathrm{e}}\left[\mathrm{cm}^{-3}\right]}{10^{17}}\right)^{0.67}
$$

Similar to $\mathrm{H}_{\beta}$, the $\mathrm{H}_{\delta}$ peak-separation, $\Delta \lambda_{\delta-\mathrm{ps}}$, in the range of $0.1-1 . \times 10^{17} \mathrm{~cm}^{-3}$ amounts to

$$
\Delta \lambda_{\delta-\mathrm{ps}}[\mathrm{nm}]=2.0\left(\frac{\mathrm{N}_{\mathrm{e}}\left[\mathrm{cm}^{-3}\right]}{10^{17}}\right)^{0.62} .
$$

In this work, density determinations are exclusively from FWHM of the first four Balmer series lines. Future work will address density determination from $\mathrm{H}_{\delta}$ peak separations that become clearly distinguishable for electron densities in the range of $0.1-1 \times 10^{17} \mathrm{~cm}^{-3}$, but for longer time delays than $275 \mathrm{~ns}$.

For time delay of $275 \mathrm{~ns}$, the FWHM for $\mathrm{H}_{\gamma}$ and $\mathrm{H}_{\delta}$ are $9.1 \pm 2 \mathrm{~nm}$ and $14.8 \pm 2 \mathrm{~nm}$, respectively. From Equations (2) and (3) one finds electron densities of $1.78 \pm 0.4 \times 10^{17} \mathrm{~cm}^{-3}$ from $\mathrm{H}_{\gamma}$ and 1.8 $\pm 0.3 \times 10^{17} \mathrm{~cm}^{-3}$ from $\mathrm{H}_{\delta}$. The estimated error bars are primarily due to errors in determining the baseline for the measured line profiles in the 24-nm spectral window selected for each Balmer series line. However, the $\mathrm{H}_{\gamma}$ and $\mathrm{H}_{\delta}$ values would extrapolate the formulas that are listed to be valid up to $1 \times 10^{17} \mathrm{~cm}^{-3}$. Yet the results would be consistent with electron densities obtained from $\mathrm{H}_{\alpha}$ and $\mathrm{H}_{\beta}$ lines [5,6], namely, $1.9 \times 10^{17} \mathrm{~cm}^{-3}$.

In the time-resolved laboratory data, there are pressure shifts that are larger than the gravitationalor Einstein- shift. Figure 6 exhibits the hydrogen beta line with the expected two peaks, one blue-shifted and one red-shifted due to pressure broadening [24]. The displayed data represent a re-examination of previously recorded spectra [7] in view of self-absorption and comparison with WD spectra.

The appearance of the $\mathrm{H}_{\beta}$ line shape is illustrated for electron densities of the order of up to $60 \times 10^{17} \mathrm{~cm}^{-3}$, i.e., $\eta \simeq 0.24 \mathrm{amg}$, or for electron density inferences from $\mathrm{H}_{\beta}$ near the estimated Inglis-Teller limit [8]. For $\tau=2 \mathrm{~ns}$, it is difficult to speak of a traditional line shape because only the central portion can be demarcated. The 2-ns line-of-sight data are recorded near the tail of the laser pulse, consequently, experimental variations are expected for the accumulated averages. Figure 6 shows the $\mathrm{H}_{\beta}$ line close to the Inglis-Teller limit [8] for $\tau=2 \mathrm{~ns}$.

Figure 7 illustrates results from fitting the summed profile using one or two Lorentzians. Fig. 7 also illustrates that the peak-separation is significantly modified or masked by contributions from lower density lines, moreover, a single Lorentzian fit (Fig. 7(b))is not adequate due to the discrepancies near the peak. With impact broadening [25] the dominant process in the wings, it appears reasonable to consider Lorentzians for fitting of the spectra.

The double Lorentzian fit, Fig. 7(a), simulates the summed profile as a superposition from two regions that show different electron density. As the plasma expands spatially and temporally, and if 


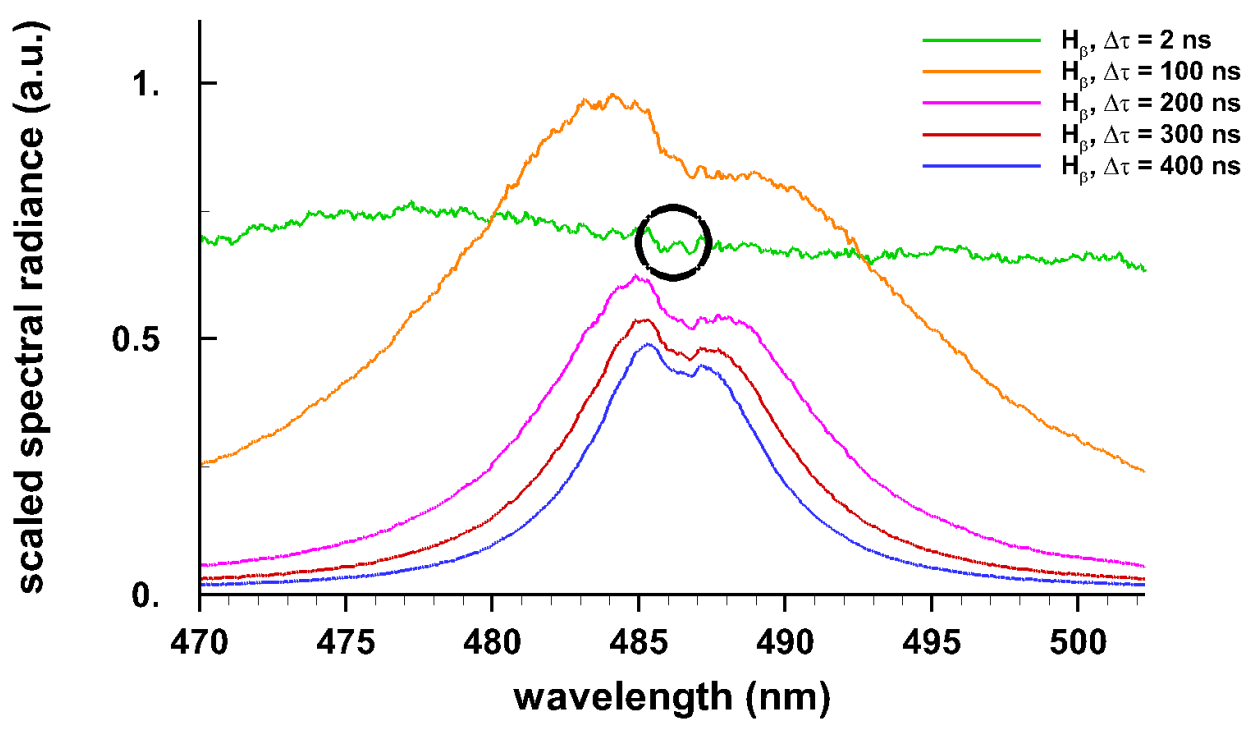

Figure 6. Line shapes of $\mathrm{H}_{\beta}$ for the indicated time delays. The encircled area in the 2-ns time-delay data shows $\mathrm{H}_{\beta}$ absorption, in other words, $\mathrm{H}_{\beta}$ laser-plasma self-absorption.

(a)

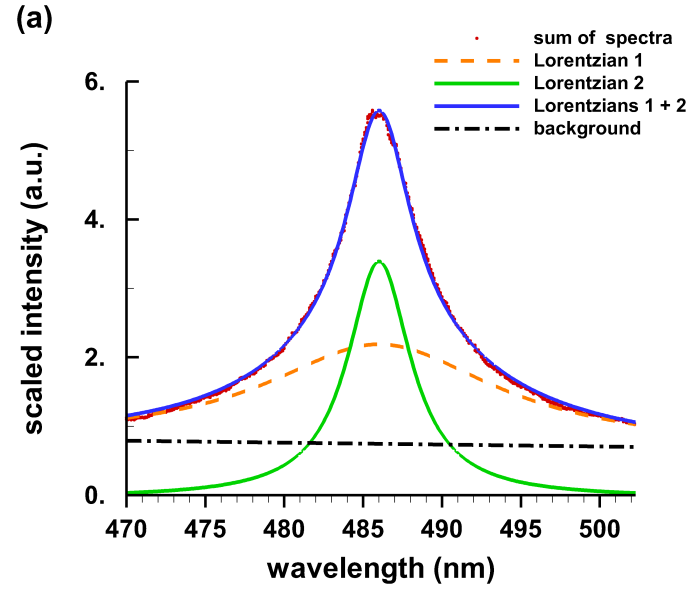

(b)

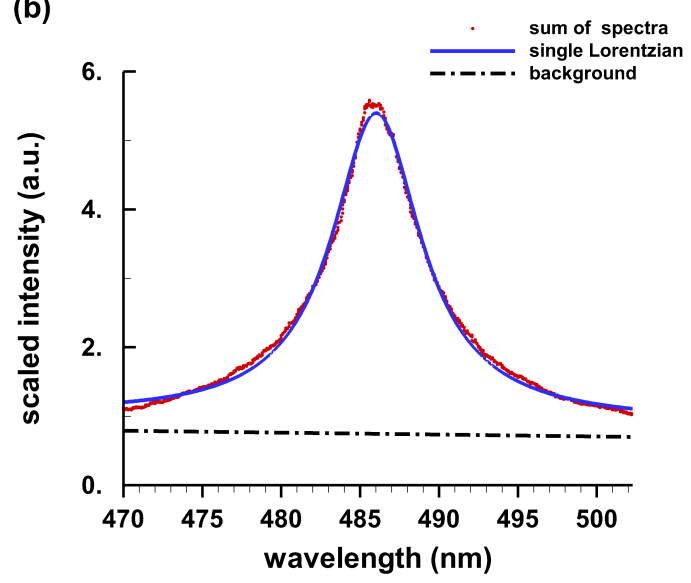

Figure 7. Fitting to summed $\mathrm{H}_{\beta}$ profiles. (a) Two Lorentzians of 4.9 and 17.6-nm widths indicating electron densities of 1.1 and $5.8 \times 10^{17} \mathrm{~cm}^{-3}$, respectively. (b) Single Lorentzian fit of 6.9-nm width and $\mathrm{N}_{\mathrm{e}}=1.8 \times 10^{17} \mathrm{~cm}^{-3}$, but with discrepancies near the wings and at center.

one were to record continuously, one would expect several regions of different density contributing to the recorded signal.

Adding the original spectra [7], recorded in the first $2.5 \mu$ s temporal window with 6-ns gate-widths, yields a result similar to using a longer gate-width in the measurement. Strictly speaking, use of gate-widths and gate-delays that entirely cover the first $2.5 \mu \mathrm{s}$, and then adding the results would be equivalent to using a $2.5 \mu$ s gate. Nevertheless, Fig. $7(b)$ is an acceptable representation of continuous recording as was the case for the collection of WD spectra.

Expanding laser-induced plasma is usually accompanied by a hypersonic shock wave, including rarefaction waves and associated temperature and density gradient regions that contribute in line-of-sight experiments. One can utilize integral inversion techniques to explore the spatial plasma 
distribution, or one can investigate line shape details in the data reduction of the spectra. In analogy with laser-ablation, occurrence of density variations can be attributed to formation of particle clusters $[26,27]$ that can be studied with flowing gas leading to percolation effects that may cause line shapes that require double-Lorentzian line shape analysis.

Self-absorption of the $\mathrm{H}_{\alpha}$ line [28] and the $\mathrm{H}_{\beta}$ line [29,30] is investigated with the so-called doubling-mirror method [31] for further evaluation of the $\mathrm{H}_{\beta}$ line electron-density diagnosis [32] . Time-resolved spectra of $\mathrm{H}_{\alpha}$ are systematically collected with 10-ns gate widths in 100-ns time-delay steps [33,34]. Laser-plasma spectra are collected in standard ambient pressure and temperature (SATP) air. Figure 8 displays measured spectra versus slit height for time delays of 300 ns, 400 ns, 700 ns, and 800-ns after intitiation of optical breakdown in air.
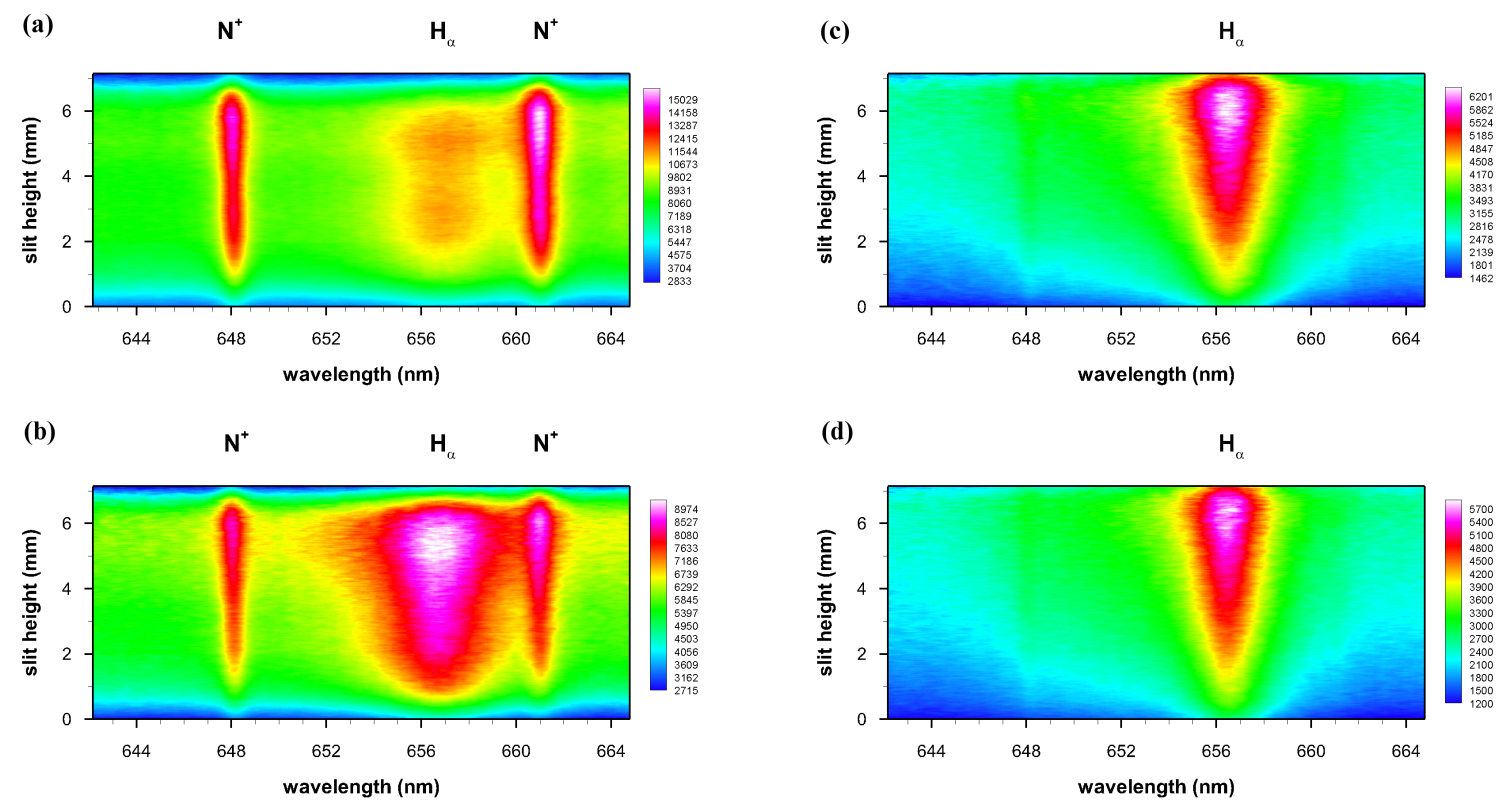

Figure 8. (a) 300 ns; (b) 400 ns time delay. (c) 700 ns; (d) 800 ns time delay. For the earlier time delays, the $\mathrm{N}^{+}$lines allow one to determine electron density and evaluate $\mathrm{H}_{\beta}$ self-absorption. The nitrogen lines are hardly discernible for later time delays in the expanding micro-plasma. Reproduced from Ref. [28] with permission.

Comparisons of data recorded with and without the doubling mirror elucidate the level of self-absorption. With respect to the indicated slit height in Figure 8, Fig. 9 illustrates Savitzky-Golay filtered spectra for $300 \mathrm{~ns}$ and $800 \mathrm{~ns}$ time delays.

(a)

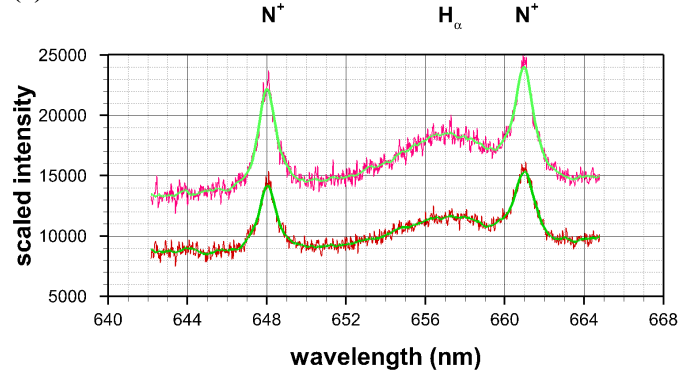

(b)

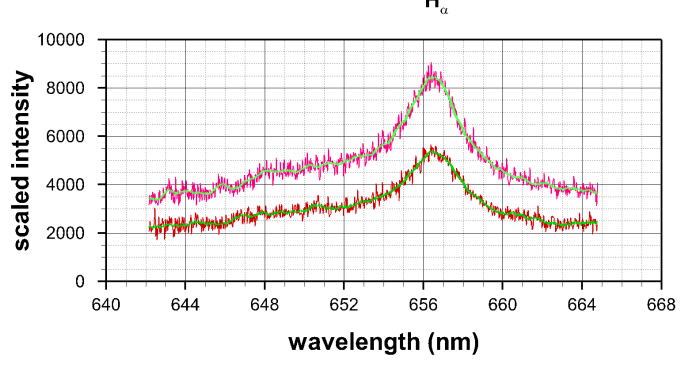

Figure 9. Comparison of spectra w/ and w/o mirror (a)) for $\mathbf{3 0 0}$ ns time delay, ((b) for 800 ns time delay. Slit height: $6.15 \mathrm{~mm}$. Reproduced from Ref. [28] with permission. 
The electron density can be determined from $\mathrm{H}_{\alpha}$ Stark broadening and shift, but it can equally be determined from ionized nitrogen $\mathrm{N}^{+}$lines for early time delays. The $\mathrm{H}_{\alpha}$ line is red-shifted from $656.28 \mathrm{~nm}$, see Fig. 9(a), but the $\mathrm{N}^{+}$lines are only slightly shifted from $648.21 \mathrm{~nm}$ and $661.06 \mathrm{~nm}$. The electron density of 14 to $20 \times 10^{17} \mathrm{~cm}^{-3}$ determined from the $\mathrm{H}_{\alpha}$ line is higher than 12 to $13 \times 10^{17} \mathrm{~cm}^{-3}$ obtained from $\mathrm{N}^{+}$for the $300 \mathrm{~ns}$ time delays. Therefore, $\mathrm{H}_{\alpha}$ shows self-absorption for delays of $300 \mathrm{~ns}$. Obviously, inferences from the FWHM of self-absorbed lines will lead to larger than true values for the electron density. However, the level of self-absorption is insignifcant for determination of electron density for time delays of $800 \mathrm{~ns}$ after optical breakdown, Fig. 9(b).

Details of the 661.06- $\mathrm{nm} \mathrm{N}^{+}$and 656.28- $\mathrm{nm}_{\alpha}$ lines reveal asymmetries that can be corrected using the doubling mirror approach [31]. The correction factor, $\mathrm{K}_{\lambda}$ or $\mathrm{K}_{\mathrm{corr}}$,

$$
\mathrm{K}_{\lambda}=\mathrm{K}_{\mathrm{corr}}=\frac{\ln \left(\mathrm{y}_{\lambda}\right)}{\mathrm{y}_{\lambda}-1}, \quad \mathrm{y}_{\lambda}=\frac{\mathrm{R}_{\lambda}-1}{\mathrm{R}_{\mathrm{C}}-1} .
$$

Analysis of the experimental data yield the ratio of the continuum radiation, $\mathrm{R}_{\mathrm{C}}$, and the signal ratio, $R_{\lambda}$, as function of wavelength, $\lambda$. Figure 10 illustrates the results.

(a)

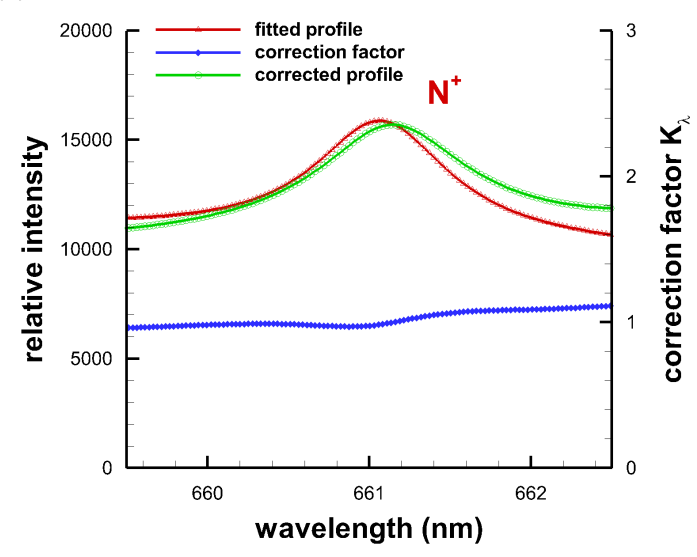

(b)

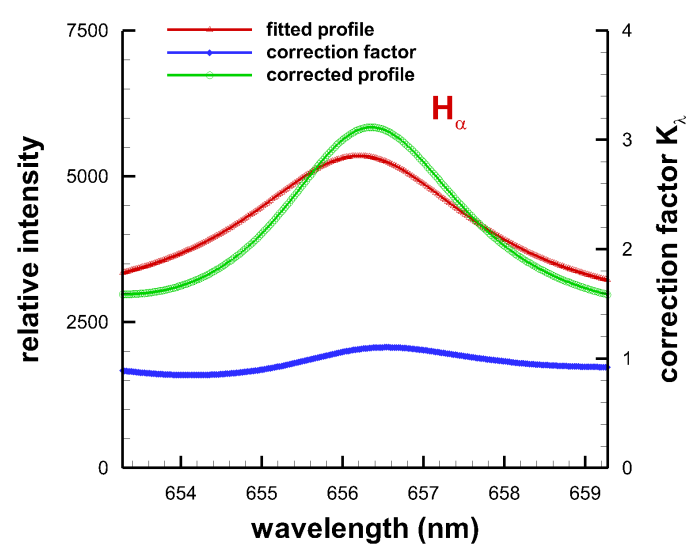

Figure 10. Corrected and fitted profile with correction factor. The fitted profile is obtained by determining the Lorentz profile from the experimental data. (a) $\mathrm{N}^{+}$for a time delay of $300 \mathrm{~ns}$, (b) $\mathrm{H}_{\alpha}$ for a time delay of $800 \mathrm{~ns}$. Slit height: $6.15 \mathrm{~mm}$. Reproduced from Ref. [28] with permission.

Additional experiments $[29,30]$ focus on the $\mathrm{H}_{\beta}$ line and for electron densities of the order of $10^{17} \mathrm{~cm}^{-3}$ using a gate width of $0.5 \mu$ s and steps of $1 \mu$ s. Figures 11(a) and 11(b) show data recorded at time delay of $5 \mu \mathrm{s}$ and $7 \mu \mathrm{s}$, respectively, from initiation of optical breakdown plasma. A plane mirror of 90 percent reflectance and a lens of 94 percent transmittance are employed to image the plasma onto itself, doubling in an ideal case the measured signal for optically thin laser-plasma.

From fitting data to the $\mathrm{H}_{\beta}$ line [29], electron densities of $0.73 \times 10^{17} \mathrm{~cm}^{-3}$ and $0.69 \times 10^{17} \mathrm{~cm}^{-3}$ are determined without and with the doubling mirror, respectively. For comparison, the N II 491.92-nm nitrogen line (measured simultaneously with $\mathrm{H}_{\beta}$ ) indicates electron densities of $0.72 \times 10^{17} \mathrm{~cm}^{-3}$ and $0.77 \times 10^{17} \mathrm{~cm}^{-3}$ without and with the doubling mirror, respectively. In addition, electron densities are determined from the $\mathrm{H}_{\beta}$ peak separation. The electron density result suggests hardly any or an insignificant amount of self-absorption. 
(a)

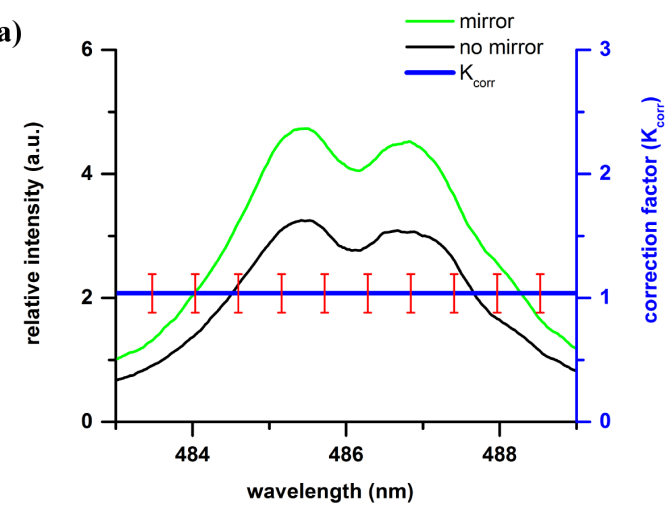

(b)

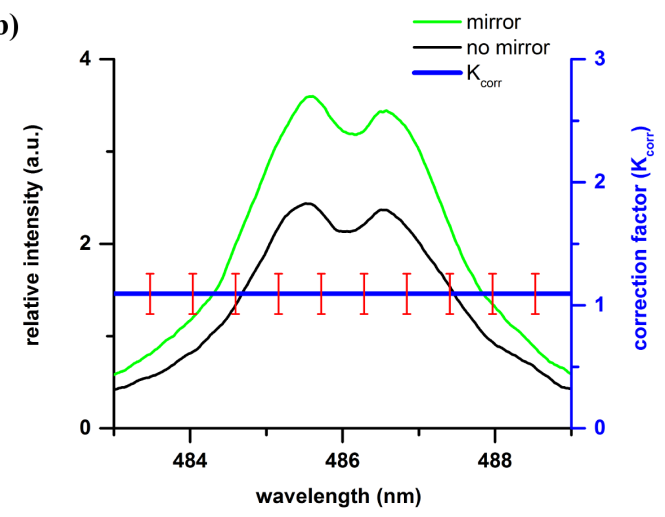

Figure 11. Measured, Savitzky-Golay filtered $\mathrm{H}_{\beta}$ air-plasma data with and without using a doubling mirror [30]. The correction factor, $\mathrm{K}_{\text {corr }}$, is almost equal to 1 . The error bars indicate the estimated errors in determination of the correction factor. Time delay (a) $5 \mu \mathrm{s}$, (b) $7 \mu \mathrm{s}$.

Recent work focuses on atomic and diatomic molecular emission spectroscopy [28]. Figures 12(a) and 12(b) illustrate line-of-sight and Abel-inverted spatial distribution of $\mathrm{CN}$ and the atomic carbon $\mathrm{C}$ I 193.09-nm line measured in second order.

(a)

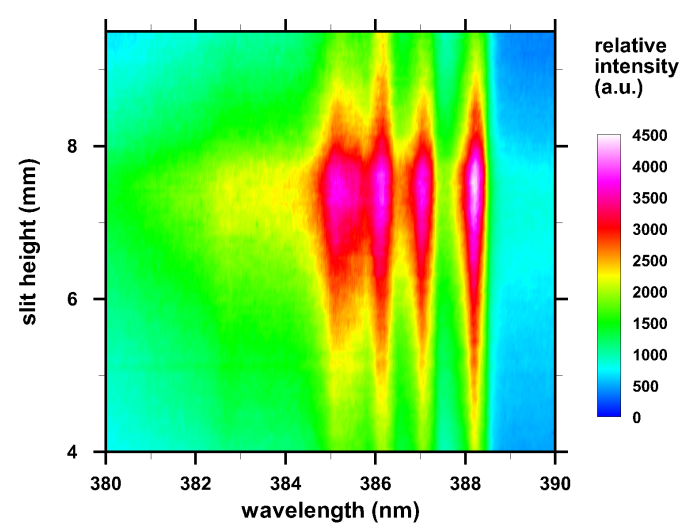

(b)

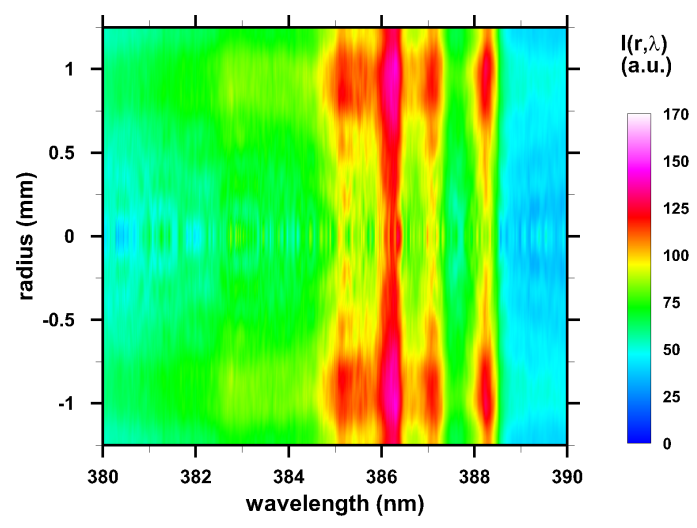

Figure 12. Diatomic molecular spectra: time delay $700 \mathrm{~ns}$, gate width $125 \mathrm{~ns}$. (a) line-of-sight contour map of measured spectra. (b) Abel inverted spectra versus radius. Near $1 \mathrm{~mm}$, the $\mathrm{CN} \Delta v=0$ sequence shows 0-0, 1-1, 2-2, 3-3 and 4-4 band heads, and the 2nd order, 193.08-nm atomic carbon line masks the 2-2 band. The $\mathrm{CN}$ signals are smaller near center. The carbon line appears narrower at the center than at the 1 -mm radial position, indicating lower electron density at center.

Plasma expansion dynamics following laser-induced optical breakdown in gases show formation of cyanide, CN, within the first few 100-ns after optical breakdown. Time-resolved, integral-inverted, line-of-sight measurements within the first microsecond reveal spatial distributions [35]. Expansion dynamics clearly affect the distribution of electrons and molecules for time delays of 700 ns, see Fig. 12(b). Abel inverted CN spectra appear nearly uniform for a time delay of $1950 \mathrm{~ns}[35,36]$.

\section{Astrophysical white dwarf spectra}

Collection of spectra from white dwarf stars preferably occurs with a resolving power sufficient for determination of the gravitational shift. Recent data for the white dwarf star GD 394 B, recorded with an echelle spectrometer on November 15, 2015, with KOA-ID: HI.20151115.19364 [3], shows a resolving power of $R=38000$, or a resolution of $\Delta \lambda=0.013 \mathrm{~nm}$. 
Figure 13(a) illustrates five overlapped regions of the recorded echelle spectra together with broad and narrow Lorentzian fits. Fig. 13(b) shows the expanded region of the central absorption. Both broad and narrow features of a white dwarf (WD), astrophysical plasma are usually understood as absorptions from an outer region of the WD photosphere. However, it can also be labelled "self-absorption" of the emitted radiation

(a)

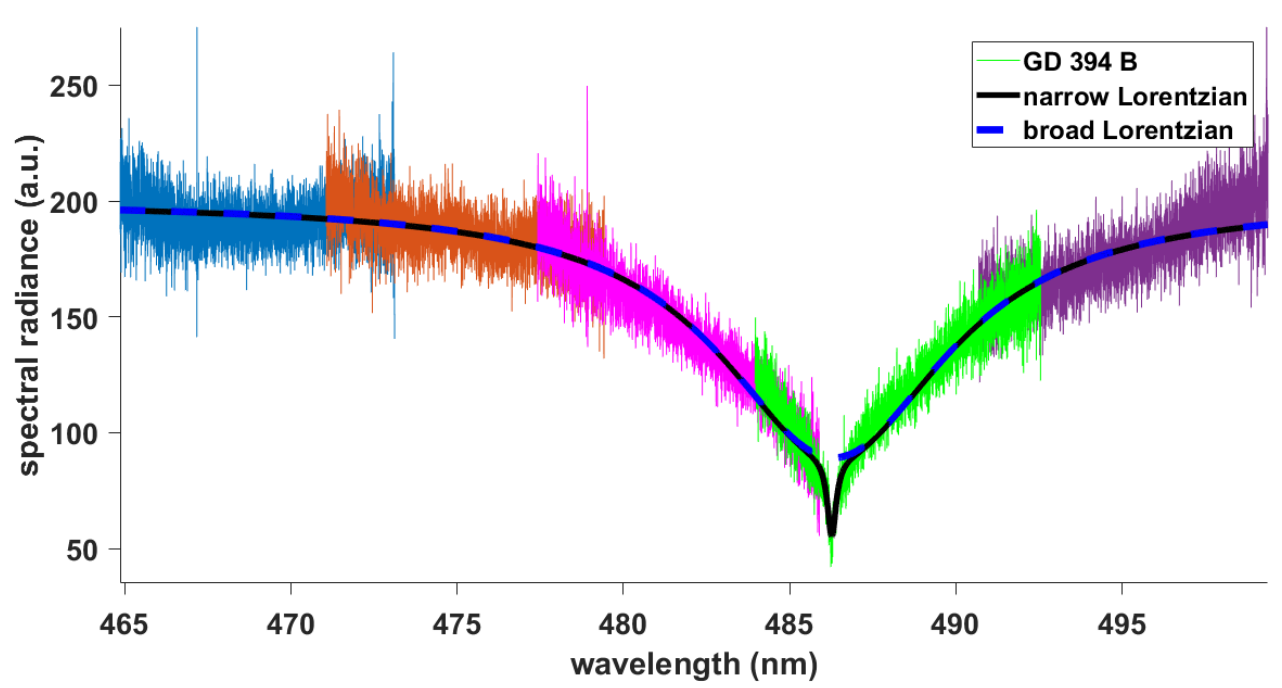

(b)

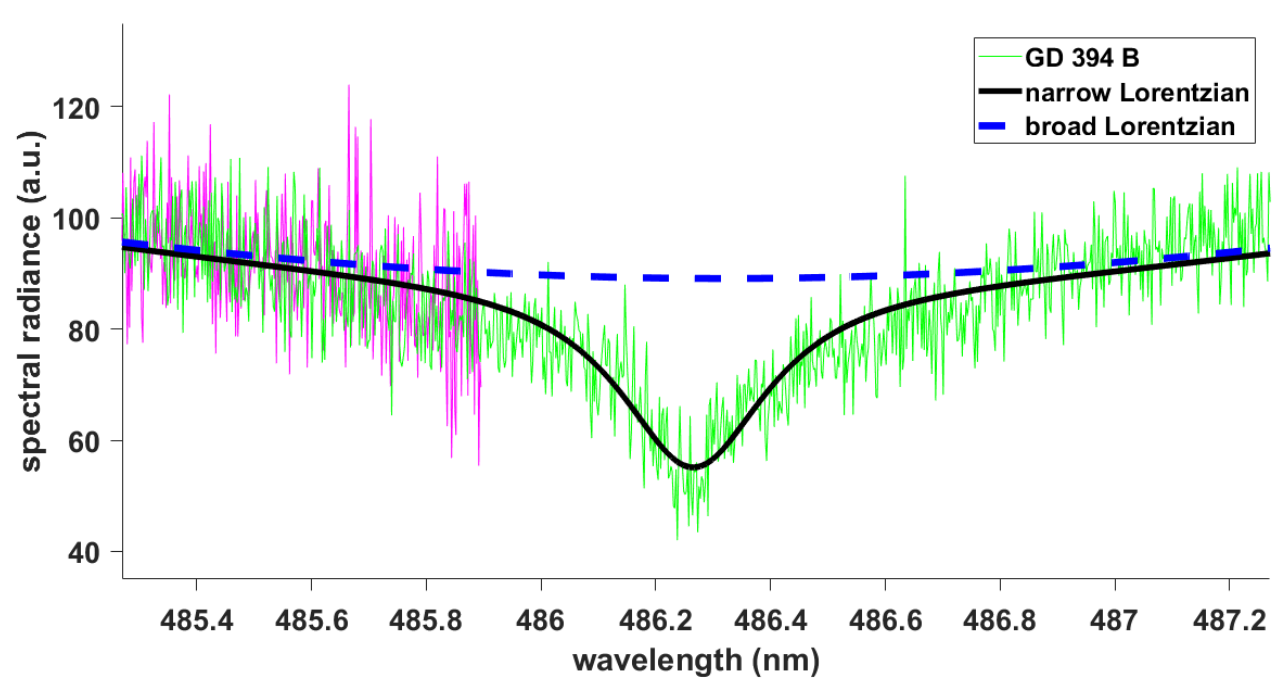

Figure 13. White dwarf GD $394 \mathrm{~B}, \mathrm{H}_{\beta}$ combined absorption profiles, recorded with the HIRES echelle spectrometer, KOA-ID: HI.20151115.19364 [3]. (a) Fitting with two Lorentzians. (b) Expanded region.

Previously communicated analysis of the GD 394 B, $\mathrm{H}_{\beta}$ spectra [37] shows narrow and broad photosphere absorption-widths of $0.39 \mathrm{~nm}$ and $7.3 \mathrm{~nm}$ that would indicate electron densities of 0.032 and $2.0 \times 10^{17} \mathrm{~cm}^{-3}$, respectively. Re-analysis of the GD 394 B white dwarf data with two-Lorentzian fits [38] and using all data points from overlapped regions of echelle orders leads to widths of $0.32 \mathrm{~nm}$ and $8.4 \mathrm{~nm}$ with corresponding electron densities of 0.022 and $2.2 \times 10^{17} \mathrm{~cm}^{-3}$, respectively. Considering the error margins for the electron density measurements from the $\mathrm{H}_{\beta}$ line [6], both results agree. Moreover, the analysis with two symmetric Lorentzians reveals 0.12-nm (average overlapped data) and 0.13-nm (use all echelle data) redshifts. Data from the same day with KOA-ID: HI.20151115.16693 and HI.20151115.17054 show fitted redshifts (average overlapped data) of $0.19 \mathrm{~nm}$ and $0.15 \mathrm{~nm}$, respectively. Figure 14 displays the spectra for the HI.20151115.17054 data, indicating a 0.18-nm redshift of the central, narrow absorption when fitting all overlapped, available data. 
(a)

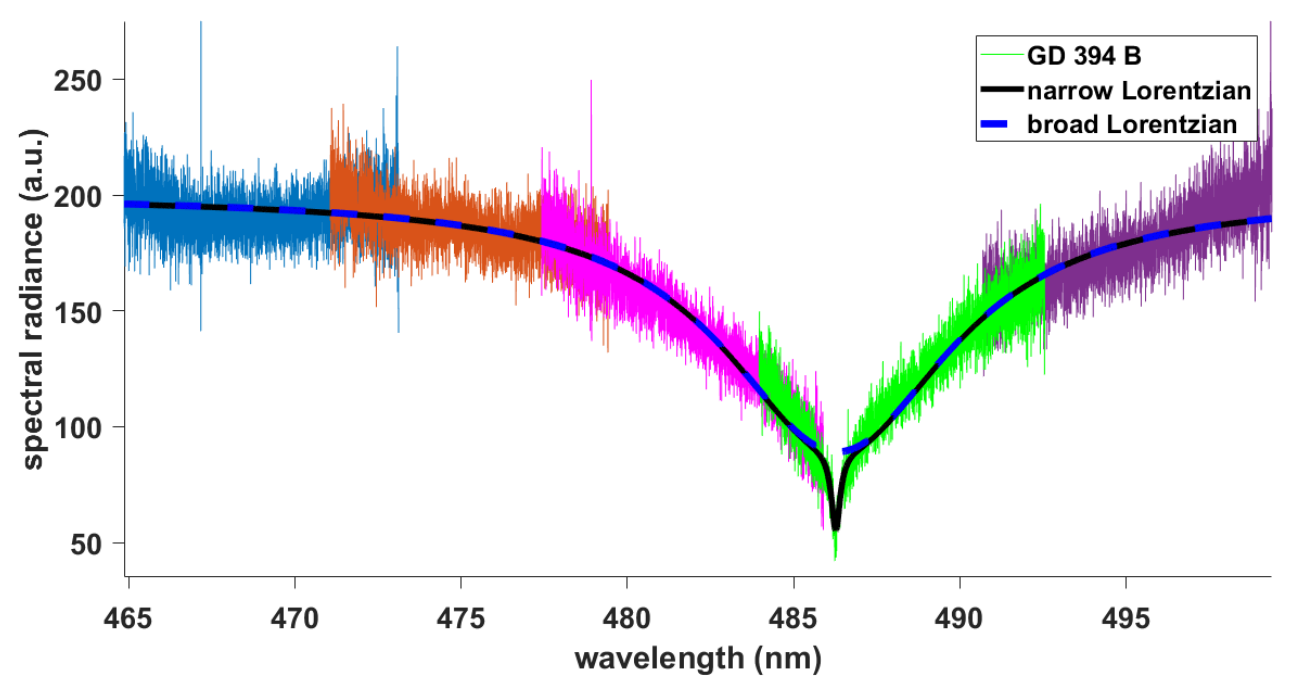

(b)

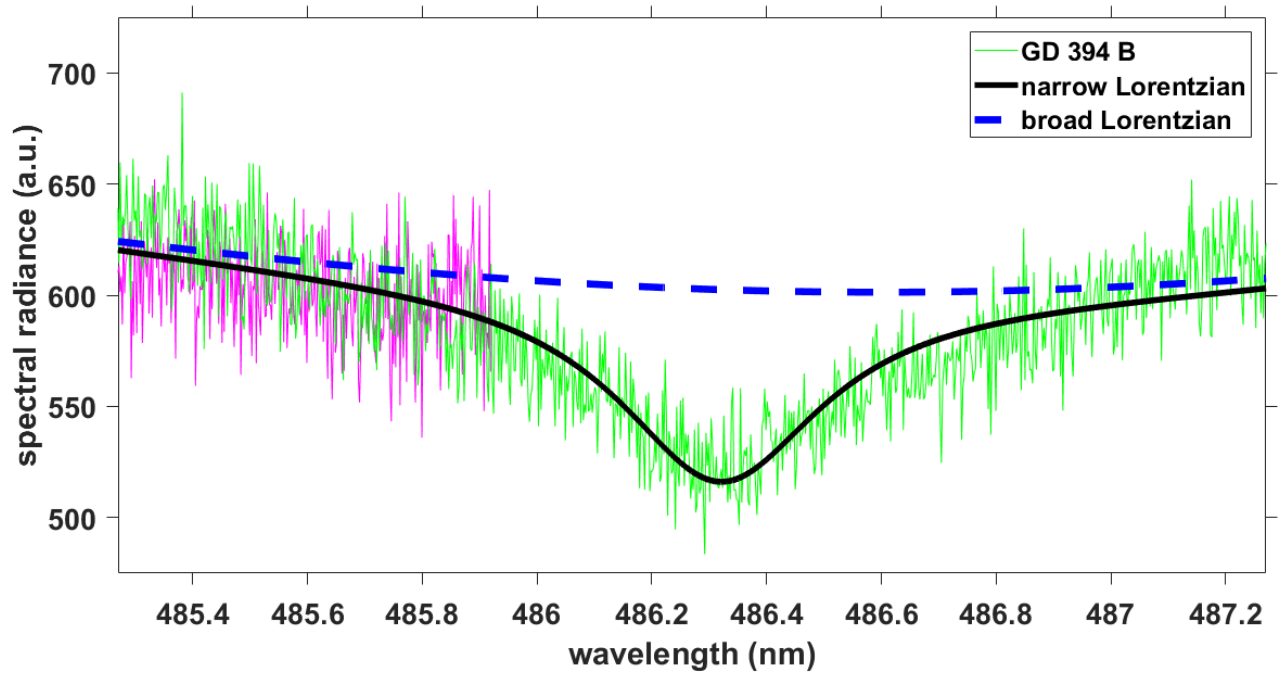

Figure 14. White dwarf GD $394 \mathrm{~B}, \mathrm{H}_{\beta}$ combined absorption profiles, recorded with the HIRES echelle spectrometer, KOA-ID: HI.20151115.17054 [3]. (a) Fitting with two Lorentzians. (b) Expanded region.

The gravitational redshift of $0.043 \mathrm{~nm}(26.77 \mathrm{~km} / \mathrm{s})$ and the photospheric component of $0.047 \mathrm{~nm}$ $(29.3 \mathrm{~km} / \mathrm{s})$ [39] account for a $0.09-\mathrm{nm}$ redshift. Further improvements in fitting with possibly asymmetric line-shapes or adjustments to the background slope could very well confirm an overall redshift of $0.09 \mathrm{~nm}$ within error bars. Analysis of the white dwarf HG 7-85 from the Hyades cluster yields consistent results $[6,40]$ for broad Lorentzian center wavelength and gravitational shifts of $0.072 \mathrm{~nm}$ and $0.08 \mathrm{~nm}$, respectively.

The comparison of laboratory and astrophysical spectra implies that different electron density regions in the atmosphere of the investigated hot $(\sim 25 \mathrm{kK})$ white dwarf stars cause absorption contributions that mask $\mathrm{H}_{\beta}$ peak separation and central dip-shifts. Molecular spectra display variations across vibrational bands, possibly indicating varying $C_{2}$ concentrations of cool $(\sim 7 \mathrm{kK})$ white dwarf atmospheres. Fitting of hydrogen spectra reveals broad and narrow profiles that may be caused by local thermodynamic non-equilibrium as WD stars cool [41,42]. The laser-induced plasma is well-reproduced in consecutive optical breakdown events allowing accumulations of several tens of laser-plasma events for each time delay. Recent laboratory measurements [43] explore white dwarf photospheric spectral lines to elucidate details in WD atmosphere modeling. 
Figure 15(a) displays a WD molecular $C_{2}$ Swan band absorption spectrum from GJ 841 B [44]. Analogous to analysis of $\mathrm{C}_{2}$ emission spectra in laser-induced plasma, the absorption spectrum in Fig. 15(a) is inverted and subsequently analyzed with the well-established diatomic molecular fitting program [45]. Fig. 15(b) illustrates fitting results for the strongest band $\Delta v=0$ of the $C_{2}$ Swan spectra.

(a)

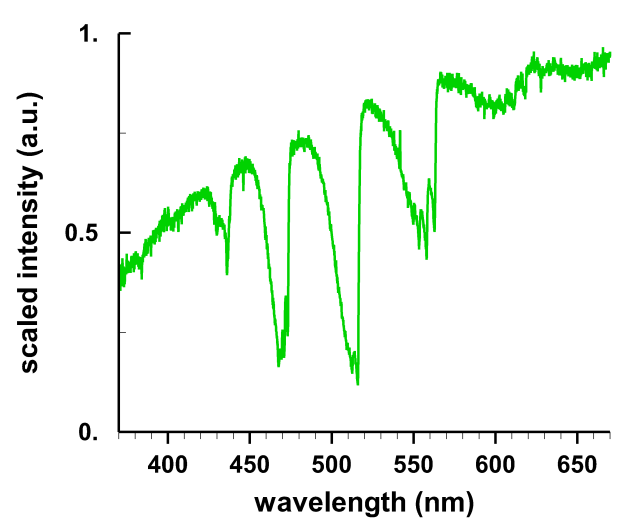

(b)
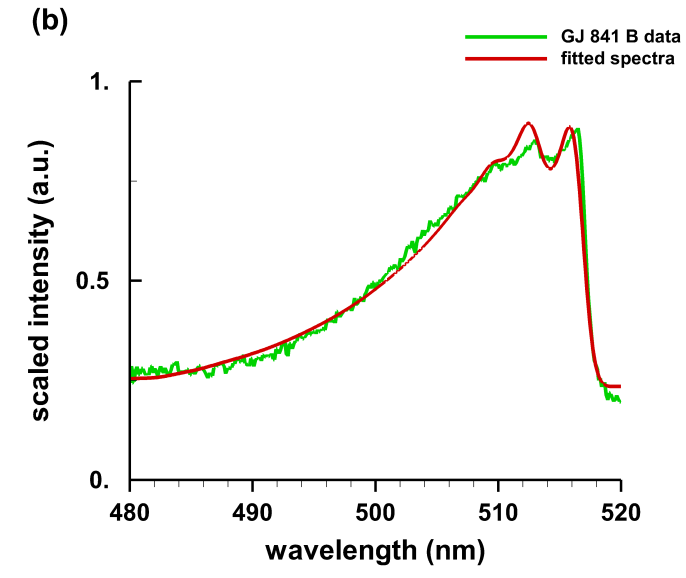

Figure 15. White dwarf GJ $841 \mathrm{~B}$ [2]. (a) $\mathrm{C}_{2}$ Swan absorption spectrum. (b) $\Delta \mathrm{v}=0: \mathrm{T}=7.1 \mathrm{kK}$, $\delta \lambda=1.8 \mathrm{~nm}$.
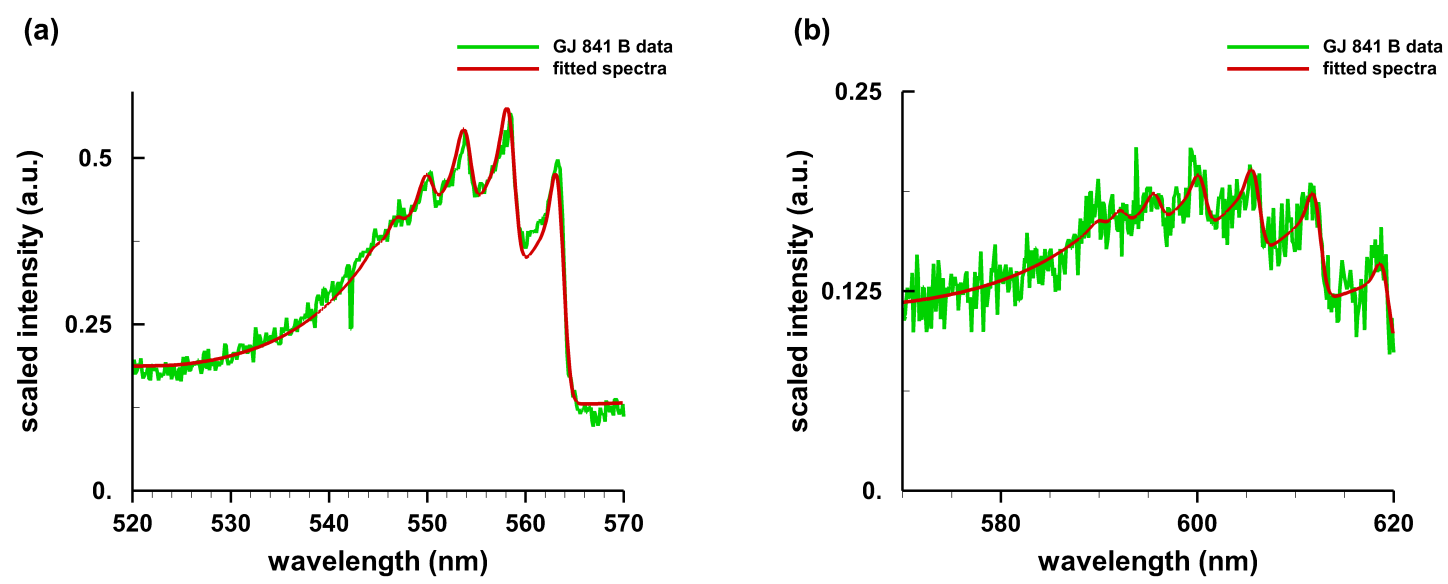

Figure 16. (a) $\Delta \mathrm{v}=-1: \mathrm{T}=4.5 \mathrm{kK}, \delta \lambda=1.5 \mathrm{~nm}$, (b) $\Delta \mathrm{v}=-2: \mathrm{T}=4.2 \mathrm{kK}, \delta \lambda=1.5 \mathrm{~nm}$.

Initial analysis indicates a temperature of $5.9 \mathrm{kK}$ and a spectral resolution, $\delta \lambda$, of $2.5 \mathrm{~nm}$ for the entire range. The recorded data are shifted by an overall $0.5 \mathrm{~nm}$ to correct for wavelength accuracy. The GJ 841 B spectra are cpatured with a resolving power of 833 , so the $0.5 \mathrm{~nm}$ adjustment is of the order of the spectral resolution. The fitting of $\Delta \mathrm{v}=+2$ and +1 indicate best-fit spectral resolutions of $1.5 \mathrm{~nm}$ that are about a factor of 2 larger than those for $\Delta \mathrm{v}=-1$ and -2 fitting. The effective temperature of the GJ $841 \mathrm{~B}$ white dwarf is documented [2] to be $\sim 7.2 \mathrm{kK}$.

The analysis of the $C_{2}$ absorption spectra suggests deviation from equilibrium due to the significant differences in computed and recorded spectra for the investigated bands. Figure 16 displays further results for the $\Delta v=-1$ and -2 bands. The extracted temperature from fitting these selected bands is lower than that from fitting all bands $\Delta v=+2,+1,0,-1,-2$. The temperature inferred from $\Delta \mathrm{v}=0$ bands is higher than that from the other bands. 


\section{Conclusions}

Atomic and molecular emission spectra of the type encountered in laser-induced breakdown spectroscopy occur in astrophysical spectra from white dwarf stars. Gas-dynamic expansion affects distribution of the laser plasma, including distributions in the plasma core and just inside the expanding shock wave. Measured hydrogen electron densities and temperatures are in the range of $1-100 \times$ $10^{17} \mathrm{~cm}^{-3}$ and $10 \mathrm{kK}$ to $120 \mathrm{kK}$ (1 to $\left.10 \mathrm{eV}\right)$, respectively. Self-absorption does not affect electron density determinations in the range of $1-10 \times 10^{17} \mathrm{~cm}^{-3}$ from $\mathrm{H}_{\alpha}$, but for electron densities in the range of $1-3 \times 10^{17} \mathrm{~cm}^{-3}$ the $\mathrm{H}_{\beta}$ line is preferred. $\mathrm{H}_{\gamma}$ and $\mathrm{H}_{\delta}$ appear well-suited for electron densities in the range of $0.1-1 . \times 10^{17} \mathrm{~cm}^{-3}$. However, laser ablation of solids reveals electron densities that are significantly higher close to the target than those encountered in gaseous breakdown plasma. Abel-inverted hydrogen and cyanide spectra indicate expansion dynamics, e.g., outgoing electron density and temperature waves. Plasma diagnosis is best accomplished with spatial and temporal resolution.

Funding: The authors appreciate the support in part by the Center for Laser Application, a State of Tennessee funded Accomplished Center of Excellence at the University of Tennessee Space Institute.

Conflicts of Interest: The authors declare no conflict of interest.

\section{References}

1. Bradt, H. Astrophysics Processes; Cambridge University Press, Cambridge, UK, 2008.

2. Dufour, P.; Blouin, S.; Coutu, S.; Fortin-Archambault, M.; Thibeault, C.; Bergeron, P.; Fontaine, G. The Montreal White Dwarf Database: A Tool for the Community. In Astronomical Society of the Pacific (ASP) Conference Series 509, Proceedings of the 20th European White Dwarf workshop, Warwick, UK, 25-29 July 2016; Tremblay, P.-E., Gaensicke, B., Marsh, T., Eds.; Utah Valley University: Orem, UT, USA, 2017; pp. 3-8, ISBN 978-1-58381-903-6. Available online: http:/ / dev.montrealwhitedwarfdatabase.org (accessed on 15 May 2019).

3. W.M. Keck Observatory Archive (KOA). Available online: https://koa.ipac.caltech.edu (accessed on 15 May 2019).

4. Kunze, H.-J. Introduction to Plasma Spectroscopy; Springer: New York, NY, USA, 2009; ISBN 978-3-642-02232-6.

5. Parigger, C.G.; Helstern, C.M.; Drake, K.A.; Gautam, G. Balmer-series hydrogen-beta line dip-shifts for electron density measurements. Int. Rev. At. Mol. Phys. 2017, 8, 53-61.

6. Parigger, C.G.; Drake, K.A.; Helstern, C.M.; Gautam, G. Laboratory Hydrogen-Beta Emission Spectroscopy for Analysis of Astrophysical White Dwarf Spectra. Atoms 2018, 6, 36.

7. Parigger, C.G.; Plemmons, D.H.; Oks, E. Balmer series H $\beta$ measurements in a laser-induced hydrogen plasma. Appl. Opt. 2003, 42, 5992-6000.

8. Inglis, D.R., Teller, E. Ionic Depression of Series Limits in One-Electron Spectra. Astrophys. J. 1939, 90, 439-448.

9. Parigger, C.G.; Swafford, L.D.; Surmick, D.M.; Witte, M.J.; Woods, A.C.; Gautam, G. Hydrogen Alpha Self-Absorption Effects in Laser-Induced Air Plasma. J. Phys.: Conf. Ser. 2014, 548012043

10. Wiese, W.L.; Kelleher, D.E. On the Cause of the Redshifts in White-Dwarf Spectra. Astrophys. J. 1971, 166, L59-L63.

11. Wiese, W.L.; Kelleher, D.E.; Paquette, D.R. Detailed Study of Stark Broadening of Balmer Lines in High-Density Plasma. Phys. Rev. A 1972, 6, 1132-1153.

12. Grabowski, B.; Halenka, J. On red shifts and asymmetries of hydrogen spectral lines. Astron. Astrophys. 1975, 45, 159-166.

13. Greenstein, J.L.; Oke, J.B.; Shipman, H.L. Effective temperature, radius, and gravitational redshift of Sirius B. Astrophys. J. 1971, 169, 563-566.

14. Holberg, J.B.; Barstow, M.A.; Bruhweiler, F.C.; Cruise, A.M.; Penny, A.J. Sirius B: A new, more accurate view. Astrophys. J. 1998, 497, 935-942.

15. Chachian M., Merches I., Radu D., Tureanu A. Electrodynamics - An Intensive Course; Springer Verlag, Heidelberg, DE 
16. Shipman, H.L.; Mehan, R.G. The unimportance of pressure shifts in the measurement of gravitational redshifts in white dwarfs. Astrophys. J. 1976, 209, 205-207.

17. Halenka, J.; Olchawa, W.; Madej, J.; Grabowski, B. Pressure shift and gravitational redshift of Balmer lines in white dwarfs: Rediscussion. Astrophys. J. 2015, 808, 131-140.

18. Falcon, R.E.; Winget, D.E.; Montgomery, M.H.; Williams, K.A. A gravitational redshift determination of the mean mass of white dwarf DA stars. Astrophys. J. 2010, 712, 585-595.

19. Griem, H.R. Plasma Spectroscopy; McGraw-Hill Book Company: New York, NY, USA, 1964.

20. Wiese WL. Spectroscopic diagnostics of low temperature plasmas: Techniques and required data. Spectrochim. Acta Part B: At. Spectrosc. 1991, 46, 831-841.

21. Cristoforetti, G., De Giacomo, A., Dell'Aglio, M., Legnaioli, S., Tognoni, E., Palleschi, V., Omenetto, N. Local thermodynamic equilibrium in laser-induced breakdown spectroscopy: Beyond the McWhirter criterion. Spectrochim. Acta Part B: At. Spectrosc. 2010, 65, 86-95.

22. Oks, E. Stark Broadening of Hydrogen and Hydrogenlike Spectral Lines in Plasmas: The Physical Insight; Alpha Science International: Oxford, UK, 2006; pp. 1-118, ISBN 1-84265-252-4.

23. Griem, H.R. Spectral Line Boradening by Plasmas; Academic Press: Cambridge, MA, USA, 1974.

24. Ivković, M.; Konjević, N.; Pavlović, Z. Hydrogen Balmer beta: The separation between line peaks for plasma electron density diagnostics and self-absorption test. J. Quant. Spectrosc. Radiat. Trans. 2015, 154, 1-8.

25. Fujimoto T. Plasma Spectroscopy; Clarendon Press: Oxford, UK, 2004.

26. Kask, N.E.; Michurin, S.V. Broadening of the spectral lines of a buffer gas and target substance. Quant. Electron. 2012, 42, 1002-1007.

27. Kask, N.E.; Lexsina, E.G.; Michurin, S.V.; Fedorov, G.M.; Chopornyak, D.B. Broadening and shift of the spectral lines of hydrogen atoms and silicon ions in laser plasma. Quant. Electron. 2015, 45, 527-532.

28. Parigger, C.G.; Gautam, G.; Woods, A.C.; Surmick, D. M.; Hornkohl, J.O. Atomic and Moleular emission spectroscopy measurements for characterization of laser-induced plsam dynamics. Trends Appl. Spectrosc. 2014, 11, 1-13.

29. Gautam, G.; Parigger, C.G.;, Surmick, D.M.; EL Sherbini, A.M. Laser plasma diagnostics and self-absorption measurements of the H $\beta$ Balmer series line. J. Quant. Spectrosc. Radiat. Transf. 2016, 170 189-193.

30. Gautam G. On Laser-induced Plasma Containing Hydrogen; (PhD Dissertation), The University of Tennessee, Knoxville, TN, USA, 2017.

31. Moon, H.-Y.; Herrera, K.K.; Omenetto, N.; Smith, B.J.; Winefordner, J.D. On the usefulness of a duplicating mirror to evaluate self-absorption effects in laser induced breakdown spectroscopy. Spectrochim. Acta Part B: At. Spectrosc. 2009, 64, 702-713.

32. Wiese, W.L. Line Broadening. In Plasma Diagnostic Techniques; Huddlestone, R.H., Leonard, S.L., Eds.; Academic Press, New York, NY, USA, 1965; pp. 265-317.

33. Parigger, C.G.; Swafford, L.D.; Surmick, D.M.; Witte M.J; Woods, A.C.; Gautam, G. Hydrogen Alpha Self-Absorption Effects in Laser-Induced Air Plasma. J. Phys.: Conf. Ser. 2014, 548, 012043.

34. Swafford, L.D.; Surmick, D.M.; Witte M.J; Woods, A.C.; Gautam, G; Parigger, C.G. Hydrogen Balmer Series Measurements in Laser-Induced Air Plasma. J. Phys.: Conf. Ser. 2014, 548, 012049.

35. Parigger, C.G; Helstern, C.M.; Gautam, G. Molecular Emission Spectroscopy of Cynide in Laser-Induced Plasma. Int. Rev. At. Mol. Phys. 2017. 8, 25-35.

36. Helstern, C.M.; Parigger, C.G. Time-resolved plasma spectroscopy of diatomic molecular cyanide. J. Phys.: Conf. Ser. 2019, in press.

37. Parigger, C.G.; Helstern, C.M.; Gautam, G; Drake, K.A. Atomic and molecular line shapes in laboratory and selected astrophysical plasma. J. Phys.: Conf. Ser. 2019, in press.

38. Keller, J. Nonlinear Fitting n-Dimensional Data with Arbitrary Functions; Mathworks File Exchange: Natick, MA, USA, 2008. Available online: https://www.mathworks.com/matlabcentral/fileexchange/ 20540-nonlinear-fitting-n-dimensional-data-with-arbitrary-functions (accessed on 22 May 2019).

39. Dickinson, N. J.; Barstow, M. A.; Welsh, B. Y.; Burleigh, M.; Farihi, J.; Redfield, S.; Unglaub, K.. The origin of hot white dwarf circumstellar features. Mon. Not. R. Astron. Soc. 2012, 423, 1397-1410.

40. Parigger, C.G.; EL Sherbini, A.M; Splinter, R. Selected laser-induced plasma spectroscopy: From medical to astrophysical applications. J. Phys.: Conf. Ser. 2019, in press.

41. Tremblay, P.-E.; Bergeron, P. Spectroscopic Analysis of DA White dwarfs: Stark broadening of hydrogen lines including nonideal effects. Astrophys. J. 2009, 696, 1755-1770. 
42. Gianninas, A.; Bergeron, P.; Dupuis, J.; Ruiz, M.T. Spectroscopic analysis of hot, hydrogen-rich white dwarf: The presence of metals and the Balmer-line problem. Astrophys. J. 2010, 720, 581-602.

43. Falcon, R.E.; Rochau ,G.A.; Bailey, J.E.; Gomez, T.A.; Montgomery, M.H.; Winget, D.E.; Nagayama, T. Laboratory measurements of white dwarf photospheric spectral lines: Hß. Astrophys. J. 2015, 806, 214-224.

44. Giammichele, N.; Bergeron, P.; Dufour, P. Know Your Neighborhood: A Detailed Model Atmosphere Analysis of Nearby White Dwarfs. Astrophys J. Suppl. Ser. 2012, 199, $29-63$.

45. Parigger, C.G.; Woods, A.C.; Surmick, D.M.; Gautam G.;Witte, M.J.; Hornkohl, J.O. Computation of diatomic molecular spectra for selected transitions of aluminum monoxide, cyanide, diatomic carbon, and titanium monoxide. Spectrochim. Acta B At. Spectrosc. 2015, 107, 132-138. 